\title{
Sistema de gestão para um hospital de referência
}

\author{
Management system for a reference hospital \\ Sistema de gestión para un hospital de referencia
}

\author{
Luana Bassetto Martin', Carmen Maria Casquel Monti Juliani", Stella Godoy Silva E Lima'II, \\ Karen Aline Batista Da Silva ${ }^{\prime v}$, Simone Cristina Paixão Dias Baptista ${ }^{V}$
}

\begin{abstract}
RESUMO
Objetivo: conhecer o perfil e demanda de urgência e emergência encaminhadas ao serviço de alta complexidade antes e após a implantação de um sistema de regulação. Metodologia: estudo retrospectivo realizado por análise estatística de relatórios administrativos do Hospital de Clinicas de Botucatu, considerados dois períodos entre 2015 e 2018. O número de pacientes do foi de 8.921 antes e 10.450 após a implantação da regulação. O estudo foi aprovado pelo Comitê de Ética em Pesquisa da instituição. Resultados: observou-se prevalência da população residente do município de Botucatu, do sexo masculino com média de idade de 40 anos, com entrada no serviço devido a fratura, trauma, afecções cardíacas e acidente vascular cerebral com tempo médio de regulação de 1 hora, 43 minutos e 48 segundos. Conclusão: encaminhamentos classificados como urgência mínima representaram $55,1 \%$ das solicitações, $12,5 \%$ dos encaminhamentos foram contrarreferenciados. 0 conhecimento do fluxo e demanda contribui para a regulação do paciente.
\end{abstract}

Descritores: Acesso aos serviços de saúde; encaminhamento e consulta; enfermagem; indicadores básicos de saúde.

\section{ABSTRACT}

Objective: to know the profile and demand of urgency and emergency referred to the high complexity service before and after the implementation of a regulation system. Methodology: retrospective study conducted by statistical analysis of administrative reports from the Hospital de Clinicas de Botucatu, considered two periods between 2015 and 2018 . The number of patients was 8,921 before and 10,450 after the implementation of regulation. The study was approved by the institution's Research Ethics Committee. Results: there was a prevalence of resident population of the municipality of Botucatu, male with a mean age of 40 years, who entered the service due to fracture, trauma, heart disease and stroke with a mean time of regulation of 1 hour, 43 minutes and 48 seconds. Conclusion: referrals classified as minimum urgency accounted for $55.1 \%$ of requests, $12.5 \%$ of referrals were counter-referenced. Knowledge of flow and demand contributes to patient regulation. Descriptors: Health services accessibility; referral and consultation; nursing; health status indicators.

\section{RESUMEN}

Objetivo: conocer el perfil y la demanda de urgencia y emergencia referidos al servicio de alta complejidad antes y después de la implementación de un sistema de regulación. Metodología: Estudio retrospectivo realizado mediante análisis estadístico de informes administrativos del Hospital de Clínicas de Botucatu, considerado dos períodos entre 2015 y 2018. El número de pacientes fue de 8.921 antes y 10.450 después de la implementación de la regulación. El estudio fue aprobado por el Comité de Ética en Investigación de la institución. Resultados: prevaleció la población residente del municipio de Botucatu, hombre con una edad media de 40 años, que ingresó al servicio por fractura, trauma, enfermedad cardíaca y accidente cerebrovascular con un tiempo medio de regulación de 1 hora 43 minutos y 48 segundos. Conclusión: Las referencias clasificadas como urgencia mínima representaron el $55.1 \%$ de las solicitudes, el $12.5 \%$ de las referencias fueron contrarreferenciadas. El conocimiento del flujo y la demanda contribuye a la regulación del paciente.

Descriptores: Accesibilidad a los servicios de salud; derivación y consulta; enfermaría; indicadores de salud.

\section{INTRODUÇÃO}

A regulação e o acesso ainda são considerados importantes desafios na constituição das Redes Regionais de Atenção à Saúde (RRAS) e na consolidação do Sistema Único de Saúde (SUS). Tais desafios são permeados pelas formas de comunicação entre os serviços, as quais contribuem no acesso do usuário.

\footnotetext{
'Enfermeira Mestre. Faculdade de Medicina de Botucatu, Universidade Estadual Paulista Júlio de Mesquita Filho. Brasil, E-mail: luana_bassetto@hotmail.com "Enfermeira. Doutora. Professora Associada. Faculdade de Medicina de Botucatu, Universidade Estadual Paulista Júlio de Mesquita Filho, Botucatu, SP, Brasil. E-mail: cjuliani@fmb.unesp.br

I'Enfermeira. Aluna do curso de doutorado. Faculdade de Medicina de Botucatu, Universidade Estadual Paulista Júlio de Mesquita Filho. Botucatu, SP, Brasil. E-mail: stella.godoy.lima@hotmail.com

IVEnfermeira. Aluna do curso de doutorado. Faculdade de Medicina de Botucatu, Universidade Estadual Paulista Júlio de Mesquita Filho. Botucatu, SP, Brasil. E-mail: karen@hcfmb.unesp.br

VEnfermeira. Aluna do curso de doutorado. Faculdade de Medicina de Botucatu, Universidade Estadual Paulista Júlio de Mesquita Filho. Botucatu, SP, Brasil. E-mail: simonepaixao@fmb.unesp.br

v'Artigo extraído da dissertação- Regulação e perfil da demanda de encaminhamentos para um hospital de referência em uma região de saúde. Programa de PósGraduação em Enfermagem da Universidade Estadual Paulista “Júlio de Mesquita Filho". Botucatu, SP, Brasil, 2019.

VIIAgradecimento à Coordenação de Aperfeiçoamento de Pessoal de Nível Superior - Brasil (CAPES - 40406008) pelo apoio financeiro.
} 
No Brasil há interdependência na saúde entre os setores público e privado'; influenciado pelo desenvolvimento tecnológico e a imprevisibilidade da ocorrência de eventos que interferem na oferta e demanda e, para equilibrar essa relação, a regulação se faz essencial².

O sistema de referência e contrarreferência na América Latina costuma possuir três níveis de atenção, o primeiro com atenção generalista e que alcança maior eficiência com a possibilidade de encaminhamentos entre primeiro, segundo e terceiro níveis de atenção, sendo que as dificuldades com as contrarreferências limitam o feedback entre os profissionais $^{3}$.

A relevância da pesquisa situa-se ao contribuir com a gestão pelo conhecimento da função da regulação em uma região de saúde. Para melhor elucidação abordaremos aspectos da regulação no Estado de São Paulo e o papel administrativo do Departamento Regional de Saúde (DRS), que é uma divisão administrativa da Secretaria de Estado de Saúde, que a torna responsável por coordenar as atividades no âmbito regional e promover a articulação intersetorial, com os municípios e organismos da sociedade civil, conforme Decreto DOE no 51.433, de 28 de dezembro de 20064 .

O Estado de São Paulo é dividido em 17 macrorregiões sendo cada uma um Departamento Regional de Saúde (DRS) numerada em algarismo romano, que agregam as regiões e organizam as ações de acordo com a complexidade na atenção à saúde. O Hospital das Clínicas (HC) faz parte do DRS-VI, composto por 5 regiões de saúde: Bauru, Avaré (Vale do Jurumirim), Jau, Lins e Botucatu (Polo Cuesta), sendo a última cidade citada a maior do Polo Cuesta, concentrando os serviços de referência dessa região ${ }^{5}$.

O objetivo principal do estudo ${ }^{\mathrm{VI}, \mathrm{VII}}$ foi conhecer o funcionamento e perfil da demanda de urgência e emergência encaminhadas a um serviço de alta complexidade antes e depois de um sistema de regulação.

\section{REVISÃO DE LITERATURA}

A Regulação em saúde é uma necessidade absoluta no Sistema Único de Saúde- SUS, por meio da qual são operadas as pactuações entre Municípios e Regiões da unidade federada ${ }^{6}$. Em agosto de 2010, com o Decreto № 56.061, a Central de Regulação de Ofertas de Serviços de Saúde do Estado de São Paulo (CROSS) foi criada pela Secretaria de Estado de Saúde, com a competência de gerenciar a regulação e disponibilizar em tempo real a situação dos Hospitais para a Regulação Pré-Hospitalar ${ }^{7}$; instituindo-se um instrumento de gestão que possibilita a otimização de recursos e resultados em saúde ${ }^{8,9}$. Posteriormente, o interior do Estado de São Paulo, em outubro de 2016 passou a funcionar com o sistema informatizado da CROSS.

As ações de regulação constituem parte importante do SUS para garantia de acesso conforme os princípios de integralidade, equidade e universalidade ${ }^{10}$. Conhecer, acompanhar e analisar o seu funcionamento torna-se de fundamental importância, pois representa a possibilidade de analisar o perfil de atendimento e demanda, para corrigir falhas e empreender as ações de acompanhamento.

Investir em sistemas de regulação consistentes, que auxiliem nos processos de gestão das vagas disponibilizadas para o SUS é fundamental, de forma que estudos sobre o tema são relevantes na conjuntura atual.

\section{MEtodologia}

Estudo retrospectivo ${ }^{11}$, desenvolvido a partir do Núcleo Interno de Regulação do HC de Botucatu, com dados referentes aos municípios do Polo Cuesta.

O Hospital é referência de média e alta complexidade para 68 municípios vinculados ao Departamento Regional de Saúde (DRS VI), dentre eles os 13 que compõem a região do Pólo Cuesta, localizado na região Centro-Oeste do estado de São Paulo e inserido na RRAS 09, objeto deste estudo ${ }^{12}$.

Para adequar o fluxo das ações de saúde de acordo com a oferta e a demanda, o SUS dispõe de central de regulação constituindo sua interface por meio do Núcleo Interno de Regulação (NIR) que delineia o perfil de complexidade e disponibiliza o recurso terapêutico adequado, de apoio diagnóstico, ambulatorial, de internação ou outros.

O serviço de regulação investigado foi implantado em 2016, com funcionamento de 24 horas e possui uma equipe da qual participam médico, enfermeiro e auxiliar administrativo.

Há três cidades: Pratânia, Areiópolis e Pereiras que não são reguladas diretamente para o HC, pois os usuários passam por atendimento secundário em outro serviço de saúde, o qual referencia para o nível terciário.

A regulação compreende a chegada da solicitação através do portal de regulação online de casos de urgência e emergência, que são analisados pelo enfermeiro regulador que aciona a especialidade requisitada e aceita o encaminhamento para avaliação ou nega as solicitações não pertinentes; apenas a origem pode cancelar a solicitação em casos de não haver mais necessidade do encaminhamento, e os médicos reguladores da CROSS podem encaminhar os pacientes como vaga zero termo que determina o encaminhamento imediato para avaliação à referência mais 
próxima, considerando a gravidade do caso (ex.1: paciente com infarto com supra e alteração de enzimas; ex.2: paciente com sinais de Acidente Vascular Cerebral (AVC) em janela; ex.3: criança com trauma crânioencefálico (TCE) apresentando sinais de alarme; ex.4: paciente vítima de acidente com politrauma e sangramento importante); neste caso são respeitadas as pactuações estabelecidas no DRS VI e não há uma lista para tal.

O que se considera vaga zero relaciona-se com a gravidade, porém não está previamente estabelecido, depende da avaliação do regulador, razão pela qual inserimos os exemplos anteriores.

A coleta compreendeu dois períodos distintos: o primeiro referente aos meses de março de 2015 a setembro de 2016 anteriores à implantação do sistema da CROSS e o segundo após sua implantação, a partir de outubro de 2016; juntamente com o sistema interno do HC-UNESP e Núcleo Interno de Regulação- NIR.

Para a composição da amostra foram feitos cálculos estatísticos com base na demanda de atendimento e a amostra foi composta aleatoriamente por meio de sorteio.

Foram utilizados Planilha Excel ${ }^{\circledR}$ e o pacote estatístico SPSS ${ }^{\circledR}$ (Statistical Package for the Social Sciences). Trabalhou-se com frequências relativa e absoluta, com realização de um teste de proporção para comparar a demanda de atendimento e distribuição das especialidades nos dois períodos de coleta de dados considerando $p<0,05$ como nível de significância.

Foi utilizado o programa R versão 2.10 .1 considerando o número de pacientes do Polo Cuesta $\mathrm{N}=8921$ (antes) e $\mathrm{N}=10450$ (após) a implantação da regulação. Obteve-se uma amostra aleatória $(\mathrm{n}=369)$ por meio de sorteio no programa R, versão 2.10.1R corrigido pela população finita, usando uma prevalência de $50 \%$ com $95 \%$ de confiança, para verificar se houve contrarreferência. A discussão foi apoiada pelo referencial teórico Regulação em Saúde no SUS ${ }^{13}$.

O projeto foi aprovado pelo Comitê de Ética em Pesquisa da Faculdade de Medicina de Botucatu (parecer 2.668.391/2018). Considerando-se este estudo quantitativo, retrospectivo e cuja coleta se deu a partir de relatórios administrativos do serviço, houve dispensa do Termo de Consentimento Livre e Esclarecido.

\section{RESULTADOS}

Antes e após a inserção da plataforma CROSS os atendimentos referentes a moradores da cidade de Botucatu representam aproximadamente $85 \%$. A faixa etária predominante foi de 15 a 65 anos com cerca de $82 \%$ e de $18 \%$ de 0 a 15 anos.

A demanda de atendimentos por cidade antes e após a inserção da plataforma é apresentada na Tabela 1, nessa ordem; com a população de cada município.

TABELA 1: Demanda de atendimento por cidade de março de 2015 a setembro de 2016 e outubro de 2016 a abril de 2018.

\begin{tabular}{lcccccc}
\hline Cidades & População & \multicolumn{2}{c}{ Mar 2015 a Set 2016 } & \multicolumn{2}{c}{ Out 2016 a Abr 2018 } & Valor de $\boldsymbol{p}$ \\
& & $\mathbf{n}$ & $\mathbf{\%}$ & $\mathbf{n}$ & $\mathbf{\%}$ & 0,2843 \\
\hline Anhembi & 6215 & 2630 & 0,75 & 2622 & 0,85 & 0,4219 \\
Areiópolis & 11020 & 2763 & 0,79 & 2733 & 0,88 & 0,0212 \\
Bofete & 9282 & 4129 & 1,17 & 3794 & 1,22 & 0,2758 \\
Botucatu & 115606 & 273649 & 77,75 & 264503 & 85,33 & 0,0096 \\
Conchas & 17638 & 4320 & 1,23 & 4418 & 1,43 & 0,515 \\
Itatinga & 23342 & 7364 & 2,09 & 7049 & 2,27 & 0,0258 \\
Laranjal Paulista & 28240 & 2377 & 0,68 & 2453 & 0,79 & 0,0352 \\
Pardinho & 5393 & 4242 & 1,21 & 4296 & 1,39 & 0,2585 \\
Pereiras & 7454 & 1790 & 0,51 & 1666 & 0,54 & 0,0001 \\
Porangaba & 9779 & 2326 & 0,66 & 2517 & 0,81 & 0,1144 \\
Pratânia & 4274 & 1815 & 0,52 & 1664 & 0,54 & $<0,0001$ \\
São Manuel & 42200 & 11649 & 3,31 & 10625 & 3,43 & $<0,0001$ \\
Torre de Pedra & 2365 & 1275 & 0,36 & 1636 & 0,53 &
\end{tabular}

Botucatu, São Manuel e Itatinga são as cidades que apresentam maior demanda de encaminhamentos. A partir do teste de proporção nota-se aumento das solicitações e atendimentos, após a inserção da CROSS. Já para as cidades: Areiópolis, Itatinga, Pratânia, Pereiras, Anhembi e Botucatu, para a especialidade de otorrinolaringologia, não apresentou diferença significativa $(p>0,05)$ como mostra a tabela 2 que aponta as especialidades que prestaram atendimento inicial no pronto socorro. A concentração de atendimentos no Pronto Socorro Adulto/ Municipal (PSA), 
Pronto Socorro Infantil (PSI) e Pronto Atendimento da Mulher/ Maternidade foi respectivamente de 40,13\%, 22,73\% e $8,38 \%$ antes da implantação da plataforma CROSS e após as concentrações foram respectivamente $41,73 \%, 21,71 \%$ e $8,04 \%$.

Importante salientar que o PSA e PSI encaminham os pacientes ao Pronto Socorro Referenciado (PS-R) localizado no hospital para atendimento com as especialidades se necessário.

TABELA 2: Distribuição das especialidades antes (março de 2015 a setembro de 2016) e após (outubro de 2016 a abril de 2018) a inserção da CROSS.

\begin{tabular}{|c|c|c|c|c|c|}
\hline \multirow[t]{2}{*}{ Especialidades } & \multicolumn{2}{|c|}{ Mar 2015 a Set 2016} & \multicolumn{2}{|c|}{ Out2016 a Abr2018 } & \multirow[t]{2}{*}{ Valor de $p$} \\
\hline & $n$ & $\%$ & $n$ & $\%$ & \\
\hline Cirurgia torácica & 895 & 0,25 & 800 & 0,23 & 0,006 \\
\hline Cirurgia cardíaca & 151 & 0,04 & 177 & 0,05 & 0,0003 \\
\hline Cirurgia geral & 9903 & 2,81 & 10015 & 2,94 & $<0,0001$ \\
\hline Cirurgia plástica & 392 & 0,11 & 435 & 0,13 & $<0,0001$ \\
\hline Cirurgia vascular & 2634 & 0,75 & 2962 & 0,87 & $<0,0001$ \\
\hline Clinica médica geral & 22856 & 6,48 & 22695 & 6,66 & $<0,0001$ \\
\hline Infectologia & 4148 & 1,18 & 4128 & 1,21 & $<0,0001$ \\
\hline Neurocirurgia & 2483 & 0,71 & 2079 & 0,61 & 0,019 \\
\hline Neuroclinica & 3608 & 1,03 & 4090 & 1,2 & $<0,0001$ \\
\hline Odontologia & 10 & 0 & 36 & 0,01 & $<0,0001$ \\
\hline Oftalmologia & 9325 & 2,65 & 9444 & 2,77 & $<0,0001$ \\
\hline Ortopedia & 10718 & 3,05 & 8723 & 2,56 & 0,004 \\
\hline Otorrinolaringologia & 6387 & 1,81 & 5070 & 1,49 & 0,3891 \\
\hline PA da mulher & 29515 & 8,38 & 27185 & 8,04 & $<0,0001$ \\
\hline Plantão generalista & 11474 & 3,26 & 6471 & 1,9 & $<0,0001$ \\
\hline Pronto Socorro Adulto & 141331 & 40,13 & 141065 & 41,73 & $<0,0001$ \\
\hline Pronto Socorro Infantil & 80039 & 22,73 & 10849 & 21,71 & $<0,0001$ \\
\hline Psiquiatria & 2808 & 0,08 & 3558 & 1,04 & $<0,0001$ \\
\hline Pronto Socorro Referenciado Pediátrico (PS-R) & 9393 & 2,67 & 10849 & 3,17 & $<0,0001$ \\
\hline Urologia & 3640 & 1,03 & 4062 & 1,19 & $<0,0001$ \\
\hline
\end{tabular}

As hipóteses diagnósticas mais presentes se referiam a: fraturas, apendicite, dor abdominal a esclarecer, angina, infarto, anemia e dispneia, em relação aos diagnósticos clínicos com maior concentração se referem às especialidades de ortopedia; cirurgia geral e clínica médica/cardiologia.

A população masculina com média de 40 anos provenientes das regiões que não passam por classificação se destacou com $57,88 \%$ e a média de tempo de regulação foi de 1 hora, 43 minutos e 48 segundos.

As solicitações contrarreferenciadas corresponderam a $12,5 \%$ da amostra e os motivos foram relacionados ao: paciente não necessitar do serviço terciário podendo estabelecer o padrão clínico na origem; necessidade de concluir a medicação prescrita na origem; paciente em cuidados paliativos e proximidade dos familiares.

O serviço de odontologia não é uma especialidade solicitada pela regulação de urgência emergência, caso haja necessidade de avaliação a especialidade solicita interconsulta, porém se o regulador considerar necessário pode solicitar uma avaliação conjunta.

Os dados posteriores são referentes ao período após implantação do sistema CROSS, uma vez que no sistema anterior estavam indisponíveis.

Foram no total 10450 solicitações reguladas, referentes à região do Polo Cuesta. A quantidade de solicitações finalizadas como negados, aceitas, canceladas e vaga zero estão descritas na Tabela 3, de acordo com os meses, após inserção da plataforma CROSS.

Houve maior busca, nesse período pelas especialidades de: oftalmologia; ortopedia e clínica médica geral.

Do total de solicitações aceitas nota-se a presença maior do público masculino; as hipóteses diagnósticas relacionadas à fratura de membros seguida de trauma, afecções cardíacas (nas quais se enquadram infarto agudo do miocárdio (IAM), bloqueio atrioventricular, bradicardia, angina instável e angina pectoris e outras) e acidente vascular cerebral (AVC), respectivamente. 
Tabela 3. Distribuição das solicitações reguladas de acordo com os meses de outubro de 2016 a abril de 2018.

\begin{tabular}{lcccc}
\hline MESES & ACEITAS & NEGADAS & CANCELADAS & VAGA ZERO \\
\hline OUT & 218 & 09 & 40 & 00 \\
NOV & 355 & 34 & 32 & 03 \\
DEZ & 388 & 16 & 26 & 04 \\
JAN & 435 & 43 & 35 & 06 \\
FEV & 428 & 52 & 42 & 14 \\
MAR & 473 & 62 & 33 & 17 \\
ABR & 407 & 51 & 28 & 16 \\
MAI & 408 & 48 & 33 & 15 \\
JUN & 372 & 57 & 27 & 12 \\
JUL & 403 & 33 & 24 & 14 \\
AGO & 134 & 34 & 24 & 19 \\
SET & 406 & 49 & 31 & 21 \\
OUT & 424 & 48 & 44 & 09 \\
NOV & 446 & 37 & 31 & 17 \\
DEZ & 517 & 67 & 21 & 18 \\
JAN & 554 & 70 & 27 & 16 \\
FEV & 498 & 71 & 36 & 10 \\
MAR & 749 & 88 & 23 & 11 \\
ABR & 1067 & 80 & 23 & 17 \\
\hline Total & 8682 & 949 & 580 & 239 \\
\hline & $(83,1 \%)$ & $(9,1 \%)$ & $(5,5 \%)$ & $(2,3 \%)$ \\
\hline
\end{tabular}

Os motivos usuais para a negativa de casos não pertinentes são: origem possui o recurso solicitado; não há necessidade de serviço terciário; caso pode ser conduzido em serviço de menor complexidade

Os motivos usais para cancelamento são: origem resolveu com recursos próprios/ locais; após reavaliação: paciente melhorou não necessita de serviço terciário e paciente procurou serviço particular;

Dos encaminhamentos decretados pela CROSS como vaga zero $77,73 \%$ foram encaminhados ao hospital no mesmo dia e até dois dias após o encerramento da ficha, e 22,27\% não chegaram por motivos desconhecidos, já que não existe um retorno para tal.

Acura-se a predominância das regiões que não passam por uma classificação numérica, de prioridade de resolução realizada pela CROSS, sendo assim considerada como zero (0) que corresponde à cor cinza não tendo um tempo definido para regulação, foram de $28,87 \%$. As cidades que não são classificadas por cores são: Torre de Pedra, Porangaba, Pardinho e Bofete.

O significado de cada número presente é interpretado da seguinte forma:

- 1: Emergência- Resolver em 30 minutos/Vermelho;

- 2: Urgência- Resolver em 3 horas/ Laranja;

- 3: Urgência de prioridade baixa- Resolver em 6 horas/ Azul e

- 4: Urgência de prioridade mínima- reavaliar o caso/ Verde.

A frequência das solicitações de outubro de 2016 a abril de 2018, segundo a classificação das cores foi de 2,69\% para os casos emergentes, $16,88 \%$ para os casos urgentes, $25,42 \%$ para os casos de urgência de prioridade baixa e $26,14 \%$ para os casos urgentes de prioridade mínima.

\section{DISCUSSÃo}

Em consonância com os resultados apresentados, estudos mostram que a causa da superlotação é composta 85\% por baixa complexidade que poderia ser atendida em nível primário e associam-se ao fator socioeconômico, apontando o uso inadequado dos serviços de urgência/emergência ${ }^{14,15}$. E que homens possuem prevalência de comportamentos que colocam sua segurança em risco, assumindo peso significativo nos perfis de morbimortalidade ${ }^{16,17}$.

Assim, é visto que a enfermagem humanística através da compreensão empática por meio da escuta ativa na rede básica propicia o encaminhamento adequado para serviços de maior complexidade e a classificação de risco proporciona maior segurança aos usuários e à equipe, respeitando o fluxo e pactuações estabelecidas ${ }^{13,18,19}$. 
De acordo com a resolução $\mathrm{n}$ - $2.077 / 14$ do conselho federal de medicina considera-se que o paciente tem por direito 24 horas para um destino clínico seja ele para alta, internação ou leito de observação nos casos de necessidade de procedimentos de enfermagem, interconsulta para especialidades e exames complementares ${ }^{20}$. 0 tempo médio de regulação para finalização das solicitações foi de 1 hora, 43 minutos e 48 segundos e a sazonalidade foi entre as especialidades de oftalmologia, clínica médica geral e ortopedia.

No Chile concluíram que a população mais velha teve a frequência de $52,29 \%$ com maior prioridade na triagem ${ }^{21}$, já no Reino Unido prova-se que os adultos mais jovens se destacam em atendimentos não urgentes sancionando com resultados obtidos ${ }^{22}$

Na Noruega analisaram as razões de entrada nos serviços de atenção primária fora de hora de emergência e, segundo a triagem, os diagnósticos apresentados foram dores abdominais as mais comuns com nível de urgência na cor amarela e as dores torácicas dominaram as classificações de cores vermelhas, dados esses que parecidos com as hipóteses apresentadas ${ }^{23}$.

O Brasil possui um Serviço de Atendimento Móvel de Urgência (SAMU) que se faz importante no auxílio do encaminhamento do paciente para serviço de complexidade adequada ${ }^{24}$. A escassez de leito hospitalar se relaciona a superlotação por encaminhamentos vaga zero e a superação desse problema envolve os níveis: federal e estadual ${ }^{25}$.

Em 2013, houve a criação dos Núcleos Internos de Regulação (NIR), através da Política Nacional de Atenção Hospitalar, o qual contribui para melhoria contínua dos fluxos, promovendo a eficiência no uso da capacidade hospitalar, porém precisa ser respaldada pelos gestores e instituições para garantia de encaminhamento adequado, respeitando as pactuações e melhorando o treinamento dos profissionais para garantir regulação eficiente ${ }^{26-28}$.

A central de regulação e a rede de referência e contrarreferência busca a regionalização cooperativa e pactuada, tornando mais eficiente o uso de recursos e qualificação de encaminhamentos ${ }^{13}$.

Conhecer o perfil da demanda encaminhada será útil para o planejamento de estratégias e intervenções que contribuem para a regulação e fluxo do usuário.

\section{CONCLUSÃO}

O público masculino se destaca na demanda de urgência/emergência, procedentes do município de Botucatu seguido por São Manuel e Itatinga; há sazonalidade entre às especialidades de oftalmologia, clínica médica, obstetrícia e pediatria.

O tempo médio de regulação foi 1 hora, 43 minutos e 48 segundos; os municípios que não passam por classificação e as solicitações com urgência de prioridade mínima corresponderam a uma frequência de 55,1\% do total e o percentual é baixo de pacientes contrarreferenciados (12,5\%).

Houve impossibilidade de quantificar as justificativas para casos negados, cancelados e encaminhados como vaga zero, uma vez que faltam informações no preenchimento do sistema, o que pode constituir um limite neste tópico de análise.

O planejamento de estratégias é imprescindível para viabilizar o fluxo de ocupação de leitos amenizando sua escassez e compreendendo a complexidade das redes de atenção a saúde, bem como respeitando o dinamismo das demandas e necessidades. O papel dos gestores é relevante para o bom funcionamento de fluxo visando à integralidade nas redes de atenção.

\section{REFERÊNCIAS}

1. Viacava F, Oliveira RAD, Carvalho CC, Languardia J, Bellido JG. SUS: Oferta, acesso e utilização de serviços de saúde nos últimos 30 anos. Ciênc. saúde coletiva. 2018 [cited 2017 Nov 12]; 23(6):1751-62. DOI: http://dx.doi.org/10.1590/141381232018236.06022018.

2. Zocratto KBF. Mercado da saúde: uma análise da oferta e demanda. Rev. MADE. 2014 [cited 2018 Nov 12]; 12: 154-64. Availabel from: http://www.revista.amde.org.br/index.php/ramde/article/view/262.

3. Fernández MA, Roo JP, Irigoyen AC, Blanco SL, Edward AB, Juárez VT. Referral and counter-referral patient management systems in latin america: care coordination mechanisms and the role of family and community medicine. Rev. bras. med. fam. comun, 2016 [cited 2017 nov 25]; 11(Suppl 2): 37-45. Available from: https://rbmfc.org.br/rbmfc/article/download/1384/805.

4. Governo Estadual (SP) Decreto no 51.433, de 28 de dezembro de 2006. Cria unidade na Coordenadoria de Regiões de Saúde, da Secretaria da Saúde, altera a denominação e dispõe sobre a reorganização das Direções Regionais de Saúde e dá providências correlatas. Diário Oficial, Estado de São Paulo, 29 dez. 2006. Seção I, p. 1. DOE-I 29/12/2006, p. 1.

5. Hospital das Clínicas - Faculdade de Medicina de Botucatu. História, os primórdios do HC. [cited 2016 Ago 10]. Available from: http://www.hcfmb.unesp.br/quem-somos/. 
6. Nascimento AAM, Damasceno AK, Silva MJ, Silva MVS, Feitoza AR. Health regulation: applicability to the concretion of the unified health system mangement pact. Cogitare enferm. 2009 [cited 2017 Nov 28]; 14(2): 346-52. Available from: https://www.nesc.ufg.br/up/19/o/texto debate prof nelson-regula o em saude.pdf.

7. Governo Estadual (SP) Decreto no 56.061, de 2 de agosto de 2010. Cria, na Coordenadoria de Serviços de Saúde, da Secretaria da Saúde, a Central de Regulação de Oferta de Serviços de Saúde- CROSS e dá providências correlatas. Diário Oficial, Estado de São Paulo, 3 ago. 2010. Seção I, p. 1. Available from: https://www.al.sp.gov.br/repositorio/legislacao/decreto/2010/decreto56061-02.08.2010.html.

8. Alves MLF, Guedes HM, Martins JCA, Chianca TCM. Reference and counter reference network for emergency care assistance in a municipality in the countryside of Minas Gerais - Brazil. Rev. Med. Minas Gerais. 2015 [cited 2018 Ago 23]; 25(4): 469-75. DOI: http://www.dx.doi.org/10.5935/2238-3182.20150110.

9. Ribas DRT. Apresentação: central de regulação de ofertas de serviços de saúde [slide], 2016; 22 slides, color. [cited 2017 Mar 07]; Available from: http://www.saude.pr.gov.br/arquivos/File/ACS/oficina rede maepr.pptx.

10. Ministério da Saúde (Br) Secretaria de Atenção à Saúde. Departamento de Regulação, Avaliação e Controle de Sistemas. Diretrizes para a implantação de complexos reguladores. Brasília, DF: Ministério da Saúde, 2006 [cited 2017 Ago 10]. Available from: http://bvsms.saude.gov.br/bvs/publicacoes/pacto saude volume6.pdf.

11. Bordalo AA. Estudo transversal e/ou longitudinal. Revista Paraense de Medicina. 2006 [cited 2018 Ago 23]; 20(4). DOI: http://scielo.iec.gov.br/pdf/rpm/v20n4/v20n4a01.pdf.

12. Fundação oncocentro de São Paulo. Caracterização da assistência oncológica nas redes regionais de atenção à saúde no estado de São Paulo. RRAS 09 - DRS Bauru (Regiões de Saúde: Vale do Jurumirim, Bauru, Jaú, Lins e Polo Cuesta). 2014 [cited 2018 Jun 16]; 6-27. Available from: http://www.saude.sp.gov.br/resources/ses/perfil/profissional-da saude/destaques//boletim assist oncol rras 6.pdf.

13. Acosta AM, Lima MADS. Frequent users of emergency services: associated factors and reasons for seeking care. Rev. latinoam. Enferm. (Online), 2015 [cited 2018 Jul 27]; 23(2): 337-44. DOI: http://dx.doi.org/10.1590/0104-1169.0072.2560.

14. Uscher-Pines L, Pines J, Kellermann A, Gillen E, Mehrotra A. Emergency department visits for nonurgent conditions: systematic literature review. Am. J. Manag. Care, 2013 [cited 2018 Mai 27]; 19(1): 47-59. Available from: https://www.ncbi.nlm.nih.gov/pubmed/23379744.

15. Arruda GO, Marcon SS. Health risk behaviors of men from the southern brazil. Texto \& contexto enferm. 2018 [cited 2018 Jul 22 ]; 27(2): e2640014. DOI: http://dx.doi.org/10.1590/0104-070720180002640014.

16. Silva AV, Santos I, Kestenberg CCF, Caldas CP, Berardinelli LMM, Silva LPS. On-call listening: an application of Humanistic Theory in the clinical nursing process. Rev. enferm. UERJ [Internet], 2018 [cited 2019 Out 31]; 26: e33586. DOI: http://dx.doi.org/10.12957/reueri.2018.33586.

17. Pagliotto LF, Souza PB, Thomazini JO, Ortega ABA, Vavra SMF. Risk classification inside an urgency and emergency unit of São Paulo countryside. Cuid. Art. Enferm. 2016 [cited 2018 Feb 12]; 10(2): 148-55. Available from: http://www.webfipa.net/facfipa/ner/sumarios/cuidarte/2016v2/148-155.pdf.

18. Gomes R, Nascimento EF, Araújo FC. Why do men use health services less than women? Explanations by men with low versus higher education. Cad. Saúde Pública. 2007 [cited 2018 Out 31]; 23(3): 565-74. DOI: http://dx.doi.org/10.1590/S0102$311 \times 2007000300015$.

19. Freitas RJM, Pereira MFA, Lima CHP, Melo JN, Oliveira KKD. Violence against nursing professionals in the embracement sector with risk classification. Rev. gaúch. Enferm. 2017 [cited 2018 Jan 28]; 38(3): e62119. DOI: http://dx.doi.org/10.1590/19831447.2017.03.62119.

20. Conselho Federal de Medicina. Resolução CFM no 2.077/14. Dispõe sobre a normatização do funcionamento dos serviços hospitalares de urgência e emergência, bem como do dimensionamento da equipe médica e do sistema de trabalho. [cited 2018 Set 09]. Available from: https://portal.cfm.org.br/images/PDF/resolucao2077.pdf

21. Granda MBM, Gutiérrez GG, Fernández MF, Jaurrieta JJS. Impact of the elderly patient in the emergency department. Rev. Esp. Geriatr. Gerontol. 2018 [cited 2018 Abr 25]; 53 (3): 145-48. DOI: https://doi.org/10.1016/i.regg.2017.08.003.

22. O'KEEFFE C, Mason S, Jacques R, Nicholl J. Characterising non-urgent users of the emergency department (ED): a retrospective analysis of routine ED data. PLoSONE. 2018 [cited 2018 Jun 19]; 13 (2). DOI: https://doi.org/10.1371/iournal.pone.0192855.

23. Raknes G, Hunskaar S. Reasons for encounter by different levels of urgency in out-of-hours emergency primary health care in norway: a cross sectional study. BMC Emergency Med. 2017 [cited 2018 Feb 19]; 17(19): 17-19. DOI: https://doi.org/10.1186/s12873-017-0129-2.

24. Oliveira GN, Oca RSC, Campanharo CRV, Okuno MFP, Lopes MCBT, Batista REA. Assessment and triage: waiting time of low severity users. Rev. Enferm. UFSM. 2016 [cited 2018 Ago 24]; 6(1): 21-28. DOI: http://dx.doi.org/10.5902/2179769218911.

25. O'DWYER G, Konder MT, Reciputti LP, Macedo C, Lopes MGM. Implementation of the Mobile Emergency Medical Service in Brazil: action strategies and structural dimension. Cad. Saúde Pública (Online). 2017 [cited 2018 Mar 24]; 33(7): e00043716. DOI: http://dx.doi.org/10.1590/0102-311x00043716.

26. Soares VS. Analysis of the internal bed regulation committees from hospitals of a southern Brazilian city. Gestão e economia em saúde - Einstein. 2017 [cited 2018 Jun 17]; 15 (3): 339-43. DOI: http://dx.doi.org/10.1590/s1679-45082017gs3878.

27. Juliani C, Macphee M, Spiri W. Brazilian specialists' perspectives on the patient referral process. Healthcare (Basel). 2017 [cited $2018 \mathrm{Jul}$ 28]; 5(4). Available from: https://www.ncbi.nlm.nih.gov/pmc/articles/PMC5371910/pdf/healthcare-05-00004.pdf.

28. Fassarella CS, Silva LD, Camerini FG, Barbieri-Figueiredo MC. Organizational indicator of safety culture in a university hospital. Rev. enferm. UERJ, Rio de Janeiro, 2019; 27:e34073. DOI: http://dx.doi.org/10.12957/reueri.2019.34073. 